\title{
Implementación del rastreo de cáncer de pulmón en la Administración de Salud de Veteranos
}

\author{
Implementation of lung cancer screening in the Veterans Health Administration
}

\begin{abstract}
Objetivos
Describir experiencias a nivel organizacional y del paciente con la implementación de un programa de rastreo anual de cáncer de pulmón (RACP) en hospitales seleccionados de la Administración de Salud de Veteranos (VHA, por Veterans Health Administration) y estimar el número de pacientes de la VHA que podrían ser candidatos para el RACP.
\end{abstract}

\section{Diseño, lugar y pacientes}

Se realizó en 8 hospitales académicos de la VHA de los EE.UU. Entre 93.033 pacientes de atención primaria que fueron evaluados con criterios de rastreo, a 2.016 pacientes se les realizó RACP entre 2013 y 2015.

\section{Medición de resultados principales}

Descripción de procesos de implementación, proporciones de pacientes que aceptaron la realización de RACP, que tuvieron hallazgos positivos en los resultados de las tomografías computadas de baja dosis (nódulos para ser seguidos o hallazgos sospechosos), que se les halló cáncer de pulmón o tuvieron hallazgos incidentales, y número estimado de pacientes de la VHA que reunieron los criterios para RACP.
Layton B y col. JAMA 2017; 317(11):1159-1166.

\section{Resultados principales}

De los 4.246 pacientes que reunieron los criterios para RACP, $2.452(57,7 \%)$ acordaron realizar rastreo, y 2.106 (2.028 hombres y 78 mujeres; edad media 64,9 [DE 5,1] años) realizaron esta práctica preventiva. Entre los ocho sitios ocurrieron amplias variaciones en los procesos y las experiencias de los pacientes. De los 2.106 pacientes rastreados, $1.257(59,7 \%)$ tuvieron nódulos; 1.184 de estos pacientes $(56,2 \%)$ requirieron rastreo, $42(2,0 \%)$ requirieron otras evaluaciones pero no se les halló cáncer, y 31 $(1,5 \%)$ tuvieron cáncer de pulmón. Una variedad de hallazgos incidentales, como enfisema, otras anormalidades pulmonares y calcificación de las arterias coronarias, fueron observados en los estudios complementarios de 857 pacientes (40,7\%).

\section{Conclusiones}

Se estima que unos 900.000 pacientes de una población total de 6,7 millones de la VHA reunieron criterios para RACP. La implementación de esta práctica preventiva en la VHA probablemente conducirá a un gran número de pacientes elegibles y requerirá esfuerzos clínicos sustanciales de los pacientes y los médicos.

\section{Comentario}

Desde que la Fuerza de Tareas Preventivas de los EE.UU. (US Preventive Services Task Force) le otorgó al RACP una recomendación de grado $B$, basándose en los hallazgos del ensayo National Lung Screening Trial publicados en 2011, el número de programas de esta práctica preventiva ha crecido significativamente en los últimos años ${ }^{1-3}$. Sin embargo estos programas se chocan con importantes cuestionamientos: el alto costo y un porcentaje para nada despreciable de falsos positivos (96\%) y de sobre-diagnóstico ${ }^{4-8}$. Estas objeciones serían aún mucho mayores en países en vías de desarrollo donde los recursos son escasos y su utilización debería destinarse a prácticas más justificadas. Este trabajo describe un programa basado en un uso intensivo de recursos que implican, además de los estudios realizados, la elaboración de guías, el empleo de coordinadores fulltime, herramientas electrónicas, seguimiento de base de datos, materiales de educación de pacientes y guías de seguimiento radiológico de nódulos. Por cada 1.000 individuos rastreados, a 10 se les diagnosticará cáncer de pulmón en estadio temprano y potencialmente curable, a 5 se les diagnosticarán cánceres incurables; 20 tendrán que realizarse estudios invasivos innecesarios (broncoscopías y toracotomías), y 550 recibirán falsas alarmas y repetirán estudios ${ }^{3}$. Se calcula que estudiando a 300 individuos de entre 55 y 75 años, fumadores importantes, anualmente durante 3 años (900 tomografías), solo uno se salvará, además de los falsos positivos y el sobre-diagnóstico ya mencionados ${ }^{8}$.

En países en vías de desarrollo, en los que los años potenciales de vida perdidos son muy altos, estudiar a poblaciones mucho mayores con seguramente una importante cantidad de comorbilidades, utilizando una alta cantidad de recursos y distrayéndolos de implementaciones de políticas muchísimo más costoefectivas es insensato. A esto habría que agregarle además, que la calidad de los servicios a realizar en nuestro medio es, en promedio, muy inferior y también muy dispar entre sí comparada con la de los establecimientos donde se realizaron estos estudios.

\section{Conclusiones del comentador}

El peor riesgo de la interpretación de estos ensayos es, sin duda, su aplicación extemporánea. $Y$ el peor escenario en nuestro medio sería que autoridades políticas sin conocimientos sean seducidas por emprendedores agresivos que promulgan el uso intensivo de altas tecnologías para beneficio, fundamentalmente económico, de unos pocos y perjuicios de todo tipo para el resto de la población.

Carlos García [ Servicio de Medicina Familiar y Comunitaria del Hospital Italiano de Buenos Aires. carlos.garcia@ hospitalitaliano.org.ar ]

García C. Implementación del rastreo de cáncer de pulmón en la Administración de Salud de Veteranos. Evid Act Pract Ambul 2017.20(3):Jul-Sep. 78. Comentado de: Kinsinger LS y col. Implementation of Lung Cancer Screening in the Veterans Health Administration. JAMA Intern Med. 2017:177(3):399-406. PMID: 28135352.

\footnotetext{
Referencias

1. Moyer VA, y col. U.S. Preventive Services Task Force. Screening for Lung Cancer: U.S. Preventive Services Task Force Recommendation Statement. Ann Intern Med [Internet]. 2014 Mar 4 [cited 2017 May 21];160(5):330-8.

2. National Lung Screening Trial Research Team. Aberle DR, y col. Reduced Lung-Cancer Mortality with Low-Dose Computed Tomographic Screening. N Engl J Med [Internet]. 2011 Aug 4 [cited 2017 May 21];365(5):395-409.

3. Redberg RF, y col. Important Questions About Lung Cancer Screening Programs When Incidental Findings Exceed Lung Cancer Nodules by 40 to 1 . JAMA Intern Med [Internet]. 2017 Mar 1 [cited 2017 May 21];177(3):311.

4. Kinsinger LS, y col. Implementation of Lung Cancer Screening in the Veterans Health Administration. JAMA Intern Med [Internet]. 2017 Mar 1 [cited 2017 May 21];177(3):399-

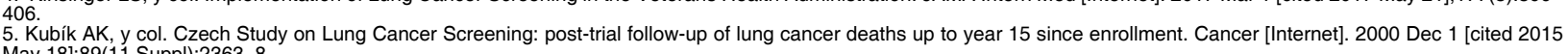

May 18];89(11 Suppl):2363-8.

8. García C. El alto costo y las verdades ocultas del exceso de tecnología dedicada al rastreo de personas asintomáticas. Evid Act Pr Amb. 2015;18(2):37-8.
} 